\title{
An insight of p-type to n-type conductivity conversion in oxygen ion-implanted ultrananocrystalline diamond films by impedance spectroscopy
}

Cite as: Appl. Phys. Lett. 110, 033102 (2017); https://doi.org/10.1063/1.4974077

Submitted: 28 September 2016 . Accepted: 03 January 2017 . Published Online: 17 January 2017

Hui Xu, Haitao Ye, David Coathup, Ivona Z. Mitrovic, Ayendra D. Weerakkody, and Xiaojun Hu
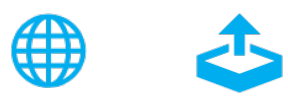

\section{ARTICLES YOU MAY BE INTERESTED IN}

Synthesis and characterization of boron doped diamond/B-SiC composite films

Applied Physics Letters 110, 031601 (2017); https://doi.org/10.1063/1.4974295

Interelectrode bridging of carbon nanotube fibrous assembly induced by gas discharge breakdown

Applied Physics Letters 110, 033101 (2017); https://doi.org/10.1063/1.4973994

On the mechanism of highly efficient $\mathrm{p}$-type conduction of Mg-doped ultra-wide-bandgap AlN nanostructures

Applied Physics Letters 110, 032102 (2017); https://doi.org/10.1063/1.4973999

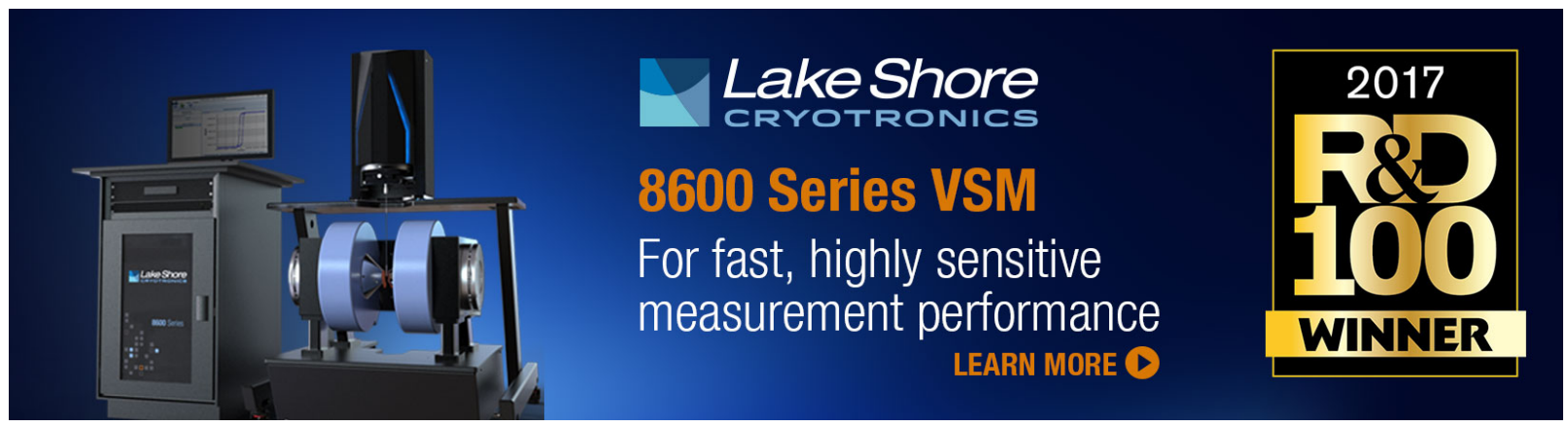




\title{
An insight of p-type to n-type conductivity conversion in oxygen ion-implanted ultrananocrystalline diamond films by impedance spectroscopy
}

\author{
Hui Xu, ${ }^{1,2}$ Haitao Ye, ${ }^{2, a)}$ David Coathup, ${ }^{2}$ Ivona Z. Mitrovic, ${ }^{3}$ Ayendra D. Weerakkody, ${ }^{3}$ \\ and Xiaojun $\mathrm{Hu}^{1, \mathrm{~b})}$ \\ ${ }^{1}$ College of Materials Science and Engineering, Zhejiang University of Technology, Hangzhou, China \\ ${ }^{2}$ School of Engineering and Applied Science, Aston University, Birmingham B4 7ET, United Kingdom \\ ${ }^{3}$ Department of Electrical Engineering and Electronics, University of Liverpool, Liverpool L69 $3 G J$, \\ United Kingdom
}

(Received 28 September 2016; accepted 3 January 2017; published online 17 January 2017)

\begin{abstract}
The impedance spectroscopy measurements were used to investigate the separated contributions of diamond grains and grain boundaries (GBs), giving an insight into p-type to n-type conductivity conversion in $\mathrm{O}^{+}$-implanted ultrananocrystalline diamond (UNCD) films. It is found that both diamond grains and GBs promote the conductivity in $\mathrm{O}^{+}$-implanted UNCD films, in which GBs make at least half contribution. The p-type conductivity in $\mathrm{O}^{+}$-implanted samples is a result of $\mathrm{H}$-terminated diamond grains, while n-type conductive samples are closely correlated with O-terminated $\mathrm{O}^{+}$implanted diamond grains and GBs in the films. The results also suggest that low resistance of GBs is preferable to obtain high mobility n-type conductive UNCD films. Published by AIP Publishing.

[http://dx.doi.org/10.1063/1.4974077]
\end{abstract}

Diamond is a promising material as a semiconductor for the fabrication of high performance electronic devices. However, the preparation of high-quality shallow-donor n-type diamond remains a challenging task. Many researchers have studied dopants including nitrogen, sulfur, oxygen, and phosphorous inserted into single and polycrystalline diamond. ${ }^{1-3}$ Studies on phosphorous doped diamond showed that the activation energy for conduction was $0.4-0.63 \mathrm{eV}$, 2,4 lower than $\sim 1.7 \mathrm{eV}$ in nitrogen doped single crystal diamond. ${ }^{5}$ Following this conclusion, room-temperature mobility up to $\sim 660 \mathrm{~cm}^{2} \mathrm{~V}^{-1} \mathrm{~s}^{-1}$ for single crystal diamond film with a phosphorus concentration of $7 \times 10^{16} \mathrm{~cm}^{-3}$ was achieved. ${ }^{4}$ Inversion channel metal-oxide-semiconductor field-effect transistors were recently fabricated based on phosphorusdoped diamond. ${ }^{6}$ However, the carriers provided to the conduction band at room temperature by phosphorus ionization are very limited with a low level of only $10^{-5}-10^{-67}$. The heavy P-doping technique was needed to induce hopping conduction, which increased the density of free carriers at room temperature for device fabrication such as junction field-effect transistors. ${ }^{8-10}$ With a high dopant concentration above $\sim 10^{20} \mathrm{~cm}^{-3}$, the conduction mechanism is described as hopping conduction with activation energy in the order of $0.05 \mathrm{eV}$ as shown by Kato et al. ${ }^{11}$

The ultrananocrystalline diamond (UNCD) film has a unique microstructure composed of nanocrystalline diamond and grain boundaries (GBs) with potential n-type doped properties. It was reported that the nitrogen doped UNCD film exhibited n-type conductivity with the mobility of $1-5 \mathrm{~cm}^{2} \mathrm{~V}^{-1} \mathrm{~s}^{-1}$ and activation energy below $10 \mathrm{meV},{ }^{12}$ which was due to the manipulation of the nanostructure of the material, leading to the enhanced $\mathrm{sp}^{2}$ regions and midgap

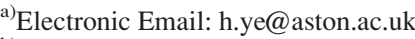

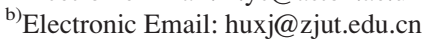

states in GBs. ${ }^{13}$ Theoretical modelling showed that nitrogen promoted $\pi$ bonded states within the GBs, resulting in an impurity band near the Fermi level. ${ }^{14}$ However, the high conductivity is required at the cost of mobilities due to the disordered nature of UNCD films. ${ }^{13}$ The nitrogen atoms tend to be located at GBs during the CVD process, seldom incorporated into diamond grains, and thus diamond grains are likely to be highly resistive in UNCD films.

Our previous work prepared both p-type and n-type conductive UNCD films by $\mathrm{O}$ ion implantation. ${ }^{15}$ A conduction model was proposed that $\mathrm{O}^{+}$-implanted diamond grains supplied n-type conductivity, while GBs gave a current path to the UNCD films. ${ }^{15}$ However, there is no direct evidence neither to distinguish the contributions of diamond grains and GBs to the conductivity of these films nor to give the insight into conductivity transformation from p-type to n-type. Impedance spectroscopy (IS) has been widely utilized to study the conduction paths within a range of conductive and less conductive materials, ${ }^{16,17}$ including polycrystalline and nanocrystalline diamond films. ${ }^{18-21}$ Its strength lies in the ability to identify the individual components contributing to the overall conductivity. Here, IS and temperature dependent current-voltage (I-V) techniques were used to obtain the inherent insight into conductivity transition in $\mathrm{O}^{+}$-implanted UNCD films.

UNCD films were deposited by a hot filament CVD system on single crystal silicon (111) wafers. The as-deposited UNCD films were treated as substrates where $\mathrm{O}$ ion implantation was performed at room temperature with $90 \mathrm{keV}$ and dose of $10^{12} \mathrm{~cm}^{-2} \cdot{ }^{15}$ For room-temperature implantations, there existed a universal critical damage density, $10^{22}$ vacancies $/ \mathrm{cm}^{3}$, which, when exceeded, resulted in the complete graphitization of heavily damaged diamond crystals upon annealing. ${ }^{22}$ It was reported that the critical dose of $\mathrm{O}$ ion implantation was $10^{16} \mathrm{~cm}^{-2}$ at room temperature with a dose 
TABLE I. The Hall effects values of the as-deposited sample, sample 900-A, and O12 series samples annealed at different temperatures.

\begin{tabular}{lcccc}
\hline \hline Sample & Sheet resistance $\left(10^{4} \Omega /\right.$ square $)$ & Hall coefficient $\left(\mathrm{m}^{2} \mathrm{C}^{-1}\right)$ & Hall mobility $\left(\mathrm{cm}^{2} \mathrm{~V}^{-1} \mathrm{~s}^{-1}\right)$ & Sheet carrier concentration $\left(10^{13} \mathrm{~cm}^{-2}\right)$ \\
\hline As-deposited & 2.24 & 39.5 & 17.6 & 1.58 \\
900-A & 0.66 & -20.6 & 31.3 & -3.03 \\
O12500 & 1.31 & 9.91 & 7.56 & 6.29 \\
O12650 & 2.30 & 2.45 & 1.07 & 25.4 \\
O12725 & 2.83 & 11.50 & 4.08 & 5.41 \\
O12800 & 3.55 & -16.30 & 4.58 & -3.84 \\
O12900 & 1.28 & -162 & 126 & -0.386 \\
O121000 & 2.43 & -19.10 & 7.86 & -3.27 \\
\hline \hline
\end{tabular}

energy of $90 \mathrm{keV}$ in UNCD films, ${ }^{23}$ which means that the damage caused by ion implantation in this work can be restored back after annealing. The implanted samples were annealed at $500,650,725,800,900$, and $1000^{\circ} \mathrm{C}$ for $30 \mathrm{~min}$ with the pressure of $4000 \mathrm{~Pa}\left(80 \% \mathrm{~N}_{2}\right.$ and $\left.20 \% \mathrm{O}_{2}\right),{ }^{15}$ named as O12500, O12650, O12725, O12800, O12900, and $\mathrm{O} 121000$, respectively. The annealing at $900{ }^{\circ} \mathrm{C}$ for $30 \mathrm{~min}$ was performed on the as-deposited UNCD film (sample 900A) to analyse the role of annealing in the electrical properties of UNCD films. Ti $(500 \mathrm{~nm}) / \mathrm{Au}(300 \mathrm{~nm})$ contacts were made on the surface of films and $\mathrm{I}-\mathrm{V}$ measurements taken at room temperature showed that ohmic-contacts formed. Hall effects measurements were performed on the samples by using the Accent HL5500 Hall system. The impedance properties of samples were determined using an AUTOLAB potentiostat-galvanostat connected with a four-probe station covering the frequency range from $0.01 \mathrm{~Hz}$ to $10 \mathrm{MHz}$. The distance between electrodes was kept constant at $2 \mathrm{~mm}$ to obtain comparable results. X-ray photoelectron spectroscopy (XPS) measurements were performed using a Kratos Axis Ultra DLD spectrometer produced by Kratos (Kratos Analytical). Temperature dependent I-V measurements were carried out by an Agilent B1500A Semiconductor Device Analyzer.

Table I lists the Hall effects data of samples. It shows that the as-deposited sample is p-type conductive while it becomes n-type conductive after $900{ }^{\circ} \mathrm{C}$ annealing with Hall mobility values increasing from 17.6 to $31.3 \mathrm{~cm}^{2} . \mathrm{V}^{-1} \mathrm{~s}^{-1}$. In $\mathrm{O}^{+}$-implanted samples, the conductivity changes from $\mathrm{p}$-type to n-type as annealing temperature $\left(\mathrm{T}_{a}\right)$ increases above

(a)

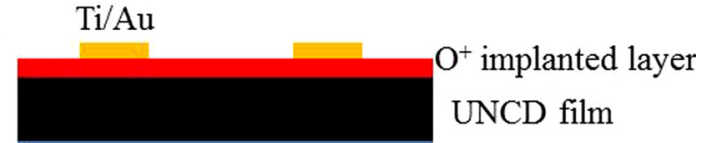

(b)

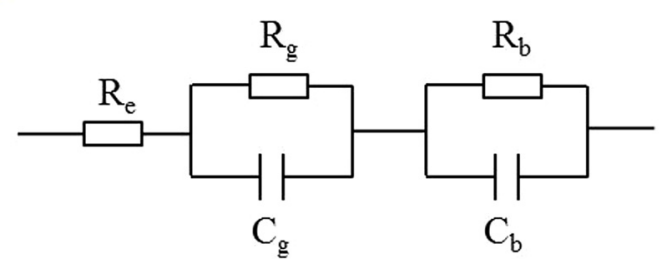

FIG. 1. (a) Schematic diagram of the cross-section of the sample. (b) Equivalent circuit for the electrical response of a UNCD sample with contributions from diamond grains (g) and grain boundaries (b), and the electrode interface (e). $800^{\circ} \mathrm{C}$. The Hall mobility values are in the range of $1-18 \mathrm{~cm}^{2}$ $\mathrm{V}^{-1} \mathrm{~s}^{-1}$ in p-type conductive samples and $4-126 \mathrm{~cm}^{2} \mathrm{~V}^{-1} \mathrm{~s}^{-1}$ in n-type samples.

IS measurements were carried out to explore the insight into the conductivity transition from p-type to n-type in $\mathrm{O}^{+}$implanted samples. The cross-section of the fabricated device is shown in Fig. 1(a), and a double resistance-capacitance (RC) parallel model in series was used to simulate the electrical conduction contribution from diamond grains and GBs, as illustrated in Fig. 1(b). The resistance between the electrode and diamond films was taken into account during practical measurements, here defined as $\mathrm{R}_{\mathrm{e}}$. Each parallel RC equivalent circuit model fitted each Cole-Cole semicircle accurately. The fitting procedure used here was the same as the one described by Kleitz and Kennedy and allowed the determination of resistance and relaxation frequencies with a good precision. ${ }^{24}$ Here, the symbols $\mathrm{R}_{\mathrm{g}}, \mathrm{R}_{\mathrm{b}}, \mathrm{C}_{\mathrm{g}}$, and $\mathrm{C}_{\mathrm{b}}$ represent the resistance $(\mathrm{R})$ and capacitance $(\mathrm{C})$ of diamond grains and GBs, respectively. The impedance as a complex number is expressed in supplementary material $\mathrm{S} 1$.

Fig. 2 shows the Cole-Cole plots measured for the silicon substrate, as-deposited sample, sample 900-A, and $\mathrm{O} 12$ series samples in the frequency range of $100 \mathrm{~Hz}-10 \mathrm{MHz}$. The inset shows the detailed Cole-Cole plot of the Si substrate, in which one semicircle is observed. The low frequency impedance value of the silicon substrate is lower

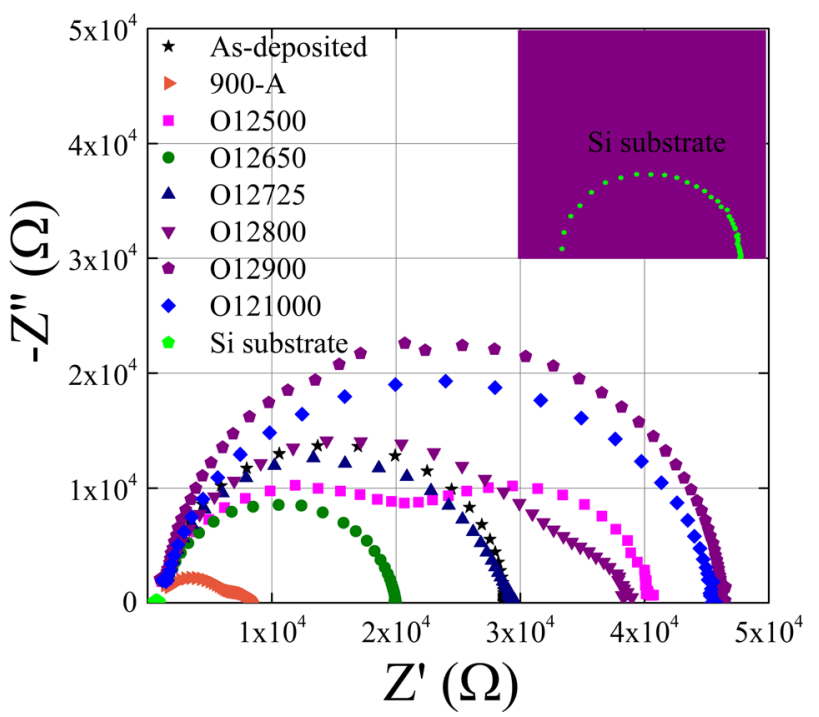

FIG. 2. The characteristic Cole-Cole plots measured for the silicon substrate, as-deposited sample, sample 900-A, and O12 series samples. The inset is the detailed Cole-Cole plot of the silicon substrate. 
than $10^{3} \Omega$, which is less than $1.5 \%$ of that of the asdeposited sample over the frequency range and thus could be ignored. The semicircular response in sample 012500 is accompanied by an additional semicircle, which extends to low frequencies, in which high frequency response is incomplete and measured as an arc due to the limited measuring range. There are also two semicircles extracted in the samples except for sample O12900, indicating that there exists two conductive paths. However, for sample O12900, one semicircle means that one conductive path plays a dominant role in the conductivity. As the microstructure of samples inevitably changes at different $\mathrm{T}_{a}$, the total impedance is required to be resolved into more refined data to analyse the contribution of each part.

In order to understand the responses quantitatively, the resistance and capacitance values were extracted according to the model shown in Fig. 1(a) and listed in Table II. It can be seen that the semicircular response at low frequency displays a capacitance value in the range of $1-9 \mathrm{nF}$, whereas the high frequency region has a capacitance of $70-110 \mathrm{pF}$. For polycrystalline materials, it is well-established that capacitance values in picofarad levels arise from the contribution to the impedance measurement from crystal grains, while nanofarad level responses can be attributed to $\mathrm{GBs} .{ }^{25}$ It is therefore clear that both nanocrystalline diamond grains and GBs in UNCD films contribute to the electrical characteristics being measured.

The $\mathrm{R}_{\mathrm{e}}$ values listed in Table II remain in the range of $1.4-1.7 \mathrm{k} \Omega$, meaning that the ohmic contact is stable and in good condition for testing. It is observed that the $R_{g}$ value of the as-deposited sample is $25.9 \mathrm{k} \Omega$, and it significantly decreases to $4.5 \mathrm{k} \Omega$ in sample 900 -A. This implies that $900^{\circ} \mathrm{C}$ annealing decreases the resistance of diamond grains in UNCD films. The $\mathrm{R}_{\mathrm{g}}$ values of samples O12500, O12650, and O12725 decrease to $19.4,15.7$, and $24.9 \mathrm{k} \Omega$ compared to that of the as-deposited sample, respectively. With $\mathrm{T}_{a}$ increasing to 800,900 , and $1000^{\circ} \mathrm{C}, \mathrm{R}_{\mathrm{g}}$ values dramatically increase to $29.2,44.6$, and $30.4 \mathrm{k} \Omega$, respectively. It is found that lowimpedance nanocrystalline diamond grains give more contribution to p-type conductive $\mathrm{O}^{+}$-implanted UNCD films while high-impedance grains promote n-type conductive films. Note that sample 900-A also exhibits n-type conduction despite the low $R_{g}$ value, indicating there is another factor promoting n-type conductivity.

Table II shows that the $R_{b}$ values are much lower than those of $R_{\mathrm{g}}$ in these samples, indicating that GBs are more

TABLE II. Resistance and capacitances values of $\mathrm{O}^{+}$-implanted UNCD films fitted from impedance spectroscopy.

\begin{tabular}{lcccccc}
\hline \hline Sample & $\mathrm{R}_{\mathrm{e}}(\mathrm{k} \Omega)$ & $\mathrm{R}_{\mathrm{g}}(\mathrm{k} \Omega)$ & $\mathrm{C}_{\mathrm{g}}(\mathrm{pF})$ & $\mathrm{R}_{\mathrm{b}}(\mathrm{k} \Omega)$ & $\mathrm{C}_{\mathrm{b}}(\mathrm{nF})$ & $\mathrm{R}_{\mathrm{g}} / \mathrm{R}_{\mathrm{b}}$ \\
\hline As-deposited & 1.4 & 25.9 & 71.4 & 1.4 & 2.2 & 18.5 \\
900-A & 1.5 & 4.5 & 105.0 & 2.2 & 7.5 & 2.0 \\
O12500 & 1.5 & 19.4 & 78.4 & 19.2 & 1.7 & 1.0 \\
O12650 & 1.4 & 15.7 & 74.9 & 2.8 & 1.5 & 5.6 \\
O12725 & 1.5 & 24.9 & 72.0 & 2.8 & 5.8 & 8.9 \\
O12800 & 1.6 & 29.2 & 79.5 & 7.6 & 3.9 & 3.8 \\
O12900 & 1.5 & 44.6 & 68.4 & $\ldots$ & $\ldots$ & $\ldots$ \\
O121000 & 1.7 & 30.4 & 94.3 & 13.0 & 8.3 & 2.3 \\
\hline \hline
\end{tabular}

conductive than diamond grains in UNCD films. The $\mathrm{R}_{\mathrm{b}}$ value of sample $900-\mathrm{A}$ is $2.2 \mathrm{k} \Omega$, slightly higher than $1.4 \mathrm{k} \Omega$ in the as-deposited sample. It reveals that annealing mainly decreases the impedance of diamond grains rather than GBs. The $\mathrm{R}_{\mathrm{b}}$ value of sample 012500 increases to $19.2 \mathrm{k} \Omega$, close to the $R_{g}$ value, which means that diamond grains and GBs almost make the equal contribution to the conductivity. Also, it is much higher than that of the as-deposited sample, indicating that the GBs become more resistive. This is probably because of the imperfection induced by ion bombardment, which cannot be recovered at low temperature annealing. The $\mathrm{R}_{\mathrm{b}}$ values are 2.8 , 2.8, 7.6, and $13.0 \mathrm{k} \Omega$ in samples $012650,012725,012800$, and O121000, respectively. The resistance of GBs is too low to be detected compared to diamond grains in sample O12900, meaning that its GBs are very conductive. Our previous work ${ }^{15}$ showed that graphene nanoribbons formed in GBs of samples 900-A and O12900 (much more formed in sample O12900), making GBs a highly conductive network. When $\mathrm{T}_{a}$ reaches $1000^{\circ} \mathrm{C}$, the conductive GB network is destroyed, leading to the increase of the impedance of GBs.

Table II also lists the ratio of $R_{g}$ to $R_{b}\left(R_{g} / R_{b}\right)$ value to estimate the conductivity contribution in UNCD films. For the as-deposited sample, this value is the highest of 18.5, which means that the GBs make the largest contribution to the conductivity. It dramatically decreases to 2.0 for sample 900-A, indicating that the contribution of diamond grains sharply increases. In samples O12500, O12650, and O12725, the $R_{g} / R_{b}$ values are 1.0, 5.6, and 8.9, respectively, which are lower than that of the as-deposited sample. The contribution of diamond grains to conductivity is increased by both diamond grains themselves and higher resistances of GBs, while it is resulted from the increasing resistances of GBs in sample 012800. It is observed that the value for sample O12900 is undetectable because of the low impedance of GBs compared to diamond grains. This implies that GBs make majority contribution to the conductivity. The $R_{g} / R_{b}$ value for sample $\mathrm{O} 121000$ is 2.3 , indicating that diamond grains provide more support in the conductivity when the nanoribbon network is destroyed.

The impedance results show that both diamond grains and GBs contribute to the conductivity in $\mathrm{O}^{+}$- implanted samples, in which GBs are more active. The contribution of diamond grains increases after implantation and annealing, except for sample O12900. It is found that low resistance of diamond grains promotes p-type conductivity while high resistance promotes n-type conductivity in $\mathrm{O}^{+}$-implanted UNCD films. It is also observed that the mobility value of sample 900-A is an order of magnitude lower than that of sample O12900, which is consistent with the resistance of diamond grains. The contribution of diamond grains also relies on the resistance of GBs in each sample, because the resistances of GBs vary after different temperature annealing. It is noted that the Hall mobility value for sample $\mathrm{O} 12900$ is $126 \mathrm{~cm}^{2} \mathrm{~V}^{-1} \mathrm{~s}^{-1}$, which is much higher than those of p-type samples. This indicates that conductive GBs help increase mobility values in n-type UNCD films more effectively than in p-type UNCD films. This is in agreement with that mobility in sample 900-A is higher than that in the asdeposited sample. The mobility value is still low in samples 
O12800 and O121000, where the resistances of GBs are much higher than that of sample 012900.

Many researchers reported that $\mathrm{H}$-terminated diamond was conductive while O-terminated diamond was insulating. ${ }^{26-28}$ To analyze the composition change of the films at different $\mathrm{T}_{a}$, we collected the $\mathrm{C} 1 \mathrm{~s}$ core-energy level spectra of the as-deposited sample, samples 900-A, O12650, and O12900 by XPS, as shown in Fig. 3. The C 1 s peaks are deconvoluted into five components at 284.8, 285.2, 286.3, 287.0 , and $288.8 \mathrm{eV}$, which are attributed to $\mathrm{sp}^{2}$ carbon, $\mathrm{sp}^{3}$ carbon, $\mathrm{C}-\mathrm{O}, \mathrm{C}=\mathrm{O}$ bonds, and $\pi-\pi^{*}$ bond, respectively. ${ }^{29-32}$ It is found that the ratio of $\mathrm{sp}^{2} \mathrm{C} / \mathrm{sp}^{3} \mathrm{C}$ is 2.67 for the asdeposited sample and slightly increases to 2.77 after $900^{\circ} \mathrm{C}$ annealing, which implies that the content of the diamond phase keeps stable. For $\mathrm{O}^{+}$-implanted samples, it becomes 2.95 for sample O12650 and 1.10 for sample O12900, indicating that the diamond content dramatically increases in sample $\mathrm{O} 12900$. The contents of $\mathrm{C}-\mathrm{O}$ and $\mathrm{C}=\mathrm{O}$ bonds of the as-deposited sample change in a small range after $900{ }^{\circ} \mathrm{C}$ annealing, from 0.09 to 0.10 and 0.07 to 0.05 , respectively, while they decrease to lower values of 0.03 and 0.02 for sample O12650. These results show that the $\mathrm{C}-\mathrm{O}$ and $\mathrm{C}=\mathrm{O}$ bonds on the surface of the as-deposited sample mainly come from the adsorbate which is removed after $650^{\circ} \mathrm{C}$ annealing. When $\mathrm{T}_{a}$ reaches $900^{\circ} \mathrm{C}$, the UNCD film is oxidized in the annealing atmosphere (the pressure of quartz tube is $4000 \mathrm{~Pa}$ with $20 \% \mathrm{O}_{2}$ remaining) and $\pi-\pi^{*}$ bonds also form. It is noted that the contents of $\mathrm{C}-\mathrm{O}$ and $\mathrm{C}=\mathrm{O}$ bonds for sample $\mathrm{O} 12900$ are 0.18 and 0.14 , higher than those of sample 900-A, suggesting that $\mathrm{O}^{+}$-ions also oxidize the film at $900^{\circ} \mathrm{C}$. The larger values of $\mathrm{C}-\mathrm{O}$ and $\mathrm{C}=\mathrm{O}$ bonds correspond to the higher amount of the $\mathrm{sp}^{3}$ carbon phase in sample O12900, which reveals that the surface of nanocrystalline diamond is terminated by oxygen. This is in agreement with impedance measurement results, where the nanocrystalline diamond grains in p-type are more conductive than those in n-type $\mathrm{O}^{+}$-implanted films. As one of the source gases during the CVD process, hydrogen makes the diamond grains

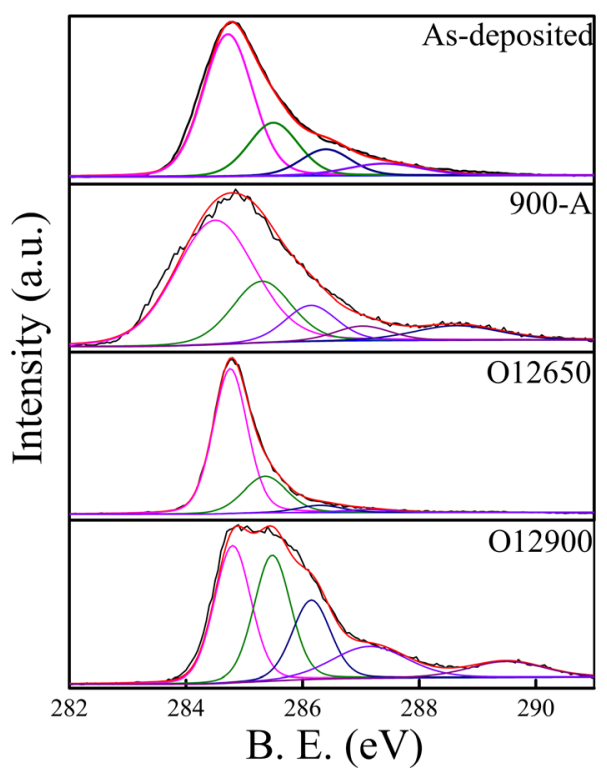

FIG. 3. Typical C 1s core-energy level spectra and their deconvolution obtained from as-deposited sample, samples 900-A, O12650, and O12900.
H-terminated and such surface induces p-type surface conductivity. ${ }^{33}$ The surface of diamond grains in $\mathrm{O}^{+}$-implanted UNCD films is oxidized to be O-terminated when $\mathrm{T}_{a}$ is above $800^{\circ} \mathrm{C}$, making the $\mathrm{R}_{\mathrm{g}}$ value higher than that of the as-deposited sample. This suggests that the transition of $\mathrm{p}$ type conductivity to n-type in $\mathrm{O}^{+}$-implanted samples is closely correlated with the diamond grains.

It had also been reported that the H-terminated surface was irreversibly destroyed after $750^{\circ} \mathrm{C}$ and above temperature annealing. ${ }^{34}$ Hydrogen bonding to the diamond grain surface and GBs in nanodiamond films was stable up to elevated temperatures of $\sim 800^{\circ} \mathrm{C}$ and then decomposed at higher temperatures. ${ }^{35}$ This is consistent with the IS result that the $\mathrm{H}$ terminated diamond surface changes to O-terminated in the $\mathrm{T}_{a}$ range of $725-800^{\circ} \mathrm{C}$. The H-terminated diamond surface had localized surface states in the band gap which would comprise the electron conductivity as they were expected to act as electron traps. ${ }^{36}$ The dramatically reduced value of the resistance of diamond grains in sample 900-A reveals the surface change of grains after $900^{\circ} \mathrm{C}$ annealing. The energy band structure at the surface of oxidized UNCD films showed a large density of states within the expected diamond band gap resulting from $\mathrm{sp}^{2}$ carbon in the GBs or other surface states related to the termination. This suggests that GBs also play an important role in the conductivity transition when hydrogen bondings decomposed from diamond grains. $\pi$ bonded states originating from GBs were reported to be responsible for the n-type conductivity in N-doped UNCD films with an activation energy below $10 \mathrm{meV} .{ }^{12}$ As the measuring temperature increases to $400^{\circ} \mathrm{C}$, the corresponding activation energy increases to $0.05-0.15 \mathrm{eV}^{37}$ It is likely that p-type conductivity is induced by the $\mathrm{H}$-terminated diamond grains, while GBs induce n-type conductivity after hydrogen bondings decomposed at higher annealing temperature, which explains the p-type to n-type conductivity conversion from the as-deposited sample to sample 900-A. However, oxygen also induced an n-type conductive layer with an activation energy of about $0.32 \mathrm{eV}$ in single crystalline diamond by ion implantation. ${ }^{38}$ It is thus not clear whether GBs or oxygen ions induce n-type conductivity in samples 012900 and O121000.

The activation energies obtained from temperature dependant I-V measurements of samples 900-A and O12900 (See supplementary material Figure S1) are $0.04 \mathrm{eV}$ and $0.11 \mathrm{eV}$, respectively, which means that the carriers in sample O12900 are more difficult to activate. This is in agreement with the IS result that the $\mathrm{R}_{\mathrm{g}}$ value of sample $\mathrm{O} 12900$ is much higher and also indicates that $\mathrm{O}$-terminated diamond grains in sample 012900 participants in the n-type conductivity. The activation energies are both lower than $0.32 \mathrm{eV}$, which means that $\pi$-bonded states with lower activation energy in these two samples also promote n-type conductivity. While the transportation of electrons largely relies on the conductivity of GBs, samples annealed at $900{ }^{\circ} \mathrm{C}$, which have lower impedance values of GBs than those samples annealed at 800 and $1000^{\circ} \mathrm{C}$, form a conductive network of electrons to obtain high mobility n-type UNCD films.

In summary, the impedance spectroscopy results directly evidence the contributions of both diamond grains and GBs to the conductivity and give an insight into conductivity 
transformation from p-type to n-type in $\mathrm{O}^{+}$-implanted UNCD films. The results show that GBs make at least half contribution. The p-type conductivity in $\mathrm{O}^{+}$-implanted samples is resulted from H-terminated diamond grains, while ntype conductive samples have a close relation to $\mathrm{O}$ terminated $\mathrm{O}^{+}$-implanted diamond grains and $\mathrm{GBs}$ in the UNCD films. The results also suggest that low resistance of GBs is preferable to obtain high mobility n-type conductive UNCD films. These UNCD films have applications in electrochemical electrodes, field emission, heterostructures, high-temperature stable ohmic contacts, and ion-sensitive field-effect transistor in harsh environment.

See supplementary material for the complex impedance of samples and temperature dependent I-V measurement results of samples 900-A and 012900.

This work was supported by the National Natural Science Foundation of China (Grant Nos. 50972129 and 50602039) and by the international science technology cooperation program of China (2014DFR51160). The work was also supported by European Union FP7 Marie Curie Action (Project No. 295208) and Horizon 2020 Action (Project No. 734578).

${ }^{1}$ I. Sakaguchi, M. N.-Gamo, Y. Kikuchi, E. Yasu, H. Haneda, T. Suzuki, and T. Ando, Phys. Rev. B 60(4), R2139 (1999).

${ }^{2}$ S. Koizumi, T. Teraji, and H. Kanda, Diamond Relat. Mater. 9(3-6), 935 (2000).

${ }^{3}$ R. Ohtani, T. Yamamoto, S. D. Janssens, S. Yamasaki, and S. Koizumi, Appl. Phys. Lett. 105(23), 232106 (2014).

${ }^{4}$ M. Katagiri, J. Isoya, S. Koizumi, and H. Kanda, Appl. Phys. Lett. 85(26), 6365 (2004).

${ }^{5}$ R. Farrer, Solid State Commun. 7(9), 685 (1969).

${ }^{6}$ T. Matsumoto, H. Kato, K. Oyama, T. Makino, M. Ogura, D. Takeuchi, T. Inokuma, N. Tokuda, and S. Yamasaki, Sci. Rep. 6, 31585 (2016).

${ }^{7}$ T. A. Grotjohn, D. T. Tran, M. K. Yaran, S. N. Demlow, and T. Schuelke, Diamond Relat. Mater. 44, 129 (2014).

${ }^{8}$ H. Kato, H. Umezawa, N. Tokuda, D. Takeuchi, H. Okushi, and S. Yamasaki, Appl. Phys. Lett. 93(20), 202103 (2008).

${ }^{9}$ K. Oyama, S.-G. Ri, H. Kato, M. Ogura, T. Makino, D. Takeuchi, N. Tokuda, H. Okushi, and S. Yamasaki, Appl. Phys. Lett. 94(15), 152109 (2009).

${ }^{10}$ M. Kasu, K. Ueda, Y. Yamauchi, A. Tallaire, and T. Makimoto, Diamond Relat. Mater. 16(4), 1010 (2007).
${ }^{11}$ H. Kato, D. Takeuchi, N. Tokuda, H. Umezawa, H. Okushi, and S. Yamasaki, Diamond Relat. Mater. 18(5-8), 782 (2009).

${ }^{12}$ O. A. Williams, S. Curat, J. E. Gerbi, D. M. Gruen, and R. B. Jackman, Appl. Phys. Lett. 85(10), 1680 (2004).

${ }^{13}$ O. A. Williams, Semicond. Sci. Technol. 21(8), R49 (2006).

${ }^{14}$ P. Zapol, M. Sternberg, L. A. Curtiss, T. Frauenheim, and D. M. Gruen, Phys. Rev. B 65(4), 045403 (2001).

${ }^{15}$ X. Hu, C. Chen, and S. Lu, Carbon 98, 671 (2016).

${ }^{16}$ A. Huanosta and A. West, J. Appl. Phys. 61(12), 5386 (1987).

${ }^{17}$ N. Hirose and A. R. West, J. Am. Ceram. Soc. 79(6), 1633 (1996).

${ }^{18}$ H. Ye, O. Gaudin, R. Jackman, P. Muret, and E. Gheeraert, Phys. Status Solidi A 199(1), 92 (2003).

${ }^{19}$ H. Ye, C. Q. Sun, H. Huang, and P. Hing, Thin Solid Films 381(1), 52 (2001).

${ }^{20} \mathrm{H}$. Ye, O. A. Williams, R. Jackman, R. Rudkin, and A. Atkinson, Phys. Status Solidi A 193(3), 462 (2002).

${ }^{21}$ M. Bevilacqua, N. Tumilty, C. Mitra, H. Ye, T. Feygelson, J. E. Butler, and R. B. Jackman, J. Appl. Phys. 107(3), 033716 (2010).

${ }^{22}$ C. Uzan Saguy, C. Cytermann, R. Brener, V. Richter, M. Shaanan, and R. Kalish, Appl. Phys. Lett. 67(9), 1194 (1995).

${ }^{23}$ X. J. Hu, J. S. Ye, H. J. Liu, Y. G. Shen, X. H. Chen, and H. Hu, J. Appl. Phys. 109(5), 053524 (2011).

${ }^{24}$ M. Kleitz, J. Kennedy, P. Vashishta, J. Mundy, and G. Shenoy, Fast Ion Transport in Solids (Elsevier, North Holland, 1979), p. 185.

${ }^{25}$ J. R. Mcdonald, Impedance Spectroscopy Emphasizing Solid Materials and Systems (Wiley, 1987).

${ }^{26}$ J. C. Piñero, D. Araújo, A. Fiori, A. Traoré, M. P. Villar, D. Eon, P. Muret, J. Pernot, and T. Teraji, Appl. Surf. Sci. 395, 200 (2016).

${ }^{27}$ T. Sakai, K.-S. Song, H. Kanazawa, Y. Nakamura, H. Umezawa, M. Tachiki, and H. Kawarada, Diamond Relat. Mater. 12(10), 1971 (2003).

${ }^{28}$ T. Maki, S. Shikama, M. Komori, Y. Sakaguchi, K. Sakuta, and T. Kobayashi, Jpn. J. Appl. Phys. 31(10A), L1446 (1992).

${ }^{29}$ Z. Chen, J. Zhao, T. Yano, T. Ooie, M. Yoneda, and J. Sakakibara, J. Appl. Phys. 88, 2305 (2000).

${ }^{30}$ L. Feng-Bin, W. Jia-Dao, C. Da-Rong, and Y. Da-Yun, Chin. Phys. B 18(5), 2041 (2009).

${ }^{31}$ D. M. Gruen, Annu. Rev. Mater. Sci. 29(1), 211 (1999).

${ }^{32}$ A. V. Sumant, P. Gilbert, D. S. Grierson, A. R. Konicek, M. Abrecht, J. E. Butler, T. Feygelson, S. S. Rotter, and R. W. Carpick, Diamond Relat. Mater. 16(4), 718 (2007).

${ }^{33}$ F. Maier, M. Riedel, B. Mantel, J. Ristein, and L. Ley, Phys. Rev. Lett. $\mathbf{8 5}(16), 3472(2000)$.

${ }^{34}$ S. Q. Lud, M. Niedermeier, P. S. Koch, P. Bruno, D. M. Gruen, M. Stutzmann, and J. A. Garrido, Appl. Phys. Lett. 96(9), 092109 (2010).

${ }^{35}$ S. Michaelson, A. Stacey, J. Orwa, A. Cimmino, S. Prawer, B. Cowie, O. A. Williams, D. Gruen, and A. Hoffman, J. Appl. Phys. 107(9), 093521 (2010).

${ }^{36}$ S. J. Sque, R. Jones, and P. R. Briddon, Phys. Rev. B 73(8), 085313 (2006).

${ }^{37}$ M. Mertens, M. Mohr, N. Wiora, K. Brühne, and H.-J. Fecht, J. Nanomater. 2015, 3 (2015).

${ }^{38}$ J. F. Prins, Phys. Rev. B 61(11), 7191 (2000). 